\title{
Pinning of Quantized Vortices in Helium Drops by Dopant Atoms and Molecules
}

\author{
Franco Dalfovo \\ Dipartimento di Matematica e Fisica, Università Cattolica, Via Musei 41, I-25121 Brescia, Italy \\ and Istituto Nazionale per la Fisica della Materia, Unità di Trento, I-38050 Povo, Italy \\ Ricardo Mayol, Martí Pi, and Manuel Barranco \\ Departament ECM, Facultat de Física, Universitat de Barcelona, E-08028 Barcelona, Spain
} (Received 22 December 1999)

\begin{abstract}
Using a density functional method, we investigate the properties of liquid ${ }^{4} \mathrm{He}$ droplets doped with atoms ( $\mathrm{Ne}$ and $\mathrm{Xe})$ and molecules $\left(\mathrm{SF}_{6}\right.$ and hydrogen cyanide). We consider the case of droplets having a quantized vortex pinned to the dopant. A liquid-drop formula is proposed that accurately describes the total energy of the complex and allows one to extrapolate the density functional results to large $N$. For a given impurity, we find that the formation of a dopant + vortex $+{ }^{4} \mathrm{He}_{N}$ complex is energetically favored below a critical size $N_{\text {cr }}$. Our results support the possibility to observe quantized vortices in helium droplets by means of spectroscopic techniques.
\end{abstract}

PACS numbers: $67.40 . V s, 33.20 . S n, 36.40 .-\mathrm{c}, 67.40 . Y v$

Since the first observation of the $\nu_{3}$ vibrational band of $\mathrm{SF}_{6}$ dissolved in ${ }^{4} \mathrm{He}$ droplets [1], the infrared spectroscopy of molecules inside or attached to helium has attracted wide interest (see, for instance, Refs. [2-4] and references therein). A major motivation for these efforts is that cold helium droplets offer the possibility of resolving rotational spectra of rather complex molecules and may constitute "the ultimate spectroscopic matrix" [5] to create and study novel species. This unique feature of helium droplets originates from their quantum nature: not only are they fluid at zero temperature due to the large zero point motion, but they also exhibit a crucial superfluid behavior. The superfluid character of ${ }^{4} \mathrm{He}$ droplets is interesting also from a fundamental viewpoint. In fact, the observation of superfluidity in finite-sized quantum systems has to do with important concepts, such as order parameter, Bose-Einstein condensation, and phase coherence, which were originally introduced for uniform systems and which are now widely used in different contexts.

In the case of liquid helium, Grebenev et al. [6] recently showed that only a rather small amount of ${ }^{4} \mathrm{He}$ atoms is needed to develop a superfluid droplet, confirming theoretical predictions [7]. In that experiment, the evidence for superfluidity is the appearance of a sharp rotational spectrum of an oxygen carbon sulfide (OCS) molecule in ${ }^{3} \mathrm{He}-{ }^{4} \mathrm{He}$ mixed drops, when the number of ${ }^{4} \mathrm{He}$ atoms surrounding the dopant [8] is larger than about 60. In the same spirit, experiments have been made to observe critical velocities [9] (i.e., the occurrence of a Landau criterion for superfluidity) and a reduction of the moment of inertia (see [10] and references therein). In contradistinction, detecting quantized vortices in He droplets still remains an open question. It is worth stressing that all these investigations have many analogies with current activity on BoseEinstein condensation in trapped gases, where new results are now available about critical velocities [11], moments of inertia [12,13], and vortices [14].
In this Letter, we address the problem of quantized vortices in liquid ${ }^{4} \mathrm{He}$ droplets. One first observes that a vortex line in a pure droplet is expected to be difficult to produce and stabilize, since it implies a significant increase of energy compared to a vortex-free droplet. To circumvent this limitation, we explore the possibility of pinning the vortex line to a dopant atom or molecule. If the dopant is deeply bound inside the droplet, it might stabilize the vortex for a time long enough to permit its detection, which is an experimental challenge. The dopant can also be used as a probe of vorticity, as both the density distortion near the pinning points and the symmetry breaking arising from the existence of a vortex line will change the effective moments of inertia of the molecule, which are measurable with spectroscopic techniques $[4,10]$. These changes are expected to be especially pronounced for linear molecules pinned along the vortex axis, whose rotation around an axis perpendicular to it will be either partially or completely impeded depending on the strength of the pinning forces.

Our purpose is to determine the energy and density profile of an impurity + vortex $+{ }^{4} \mathrm{He}_{N}$ complex for droplets up to $N=1000$, using a finite-range density functional. We then subtract from its energy that of the same droplet without vortex and/or impurity and show that the difference fits very well a liquid-drop formula, which allows one to safely extrapolate to larger droplets. We use the Orsay-Paris (OP) functional [15], based on an effective nonlocal interaction with a few parameters fixed to reproduce known properties of bulk liquid He. This functional has been shown to accurately reproduce the static properties of pure and doped He clusters [16] and has also been used to describe a quantized vortex line in bulk liquid helium [17]. In the latter case, the vortex is included with the Feynman-Onsager ansatz, i.e., by adding an extra centrifugal energy associated with the velocity field, which is singular on the vortex axis, thus forcing the density to vanish. At zero temperature this approximation is a 
reasonable starting point, since it enormously reduces the computational effort (for a recent discussion about its accuracy, see [18]). For doped droplets, one has also to include the helium-molecule interaction, which acts as an external potential in which the helium density adjusts to minimize the energy. Potentials for rare gas impurities have been taken from [19], that of the spherically averaged $\mathrm{SF}_{6}$ from [20], and that of hydrogen cyanide ( $\left.\mathrm{HCN}\right)$ from [21]. The total energy is thus written as

$$
E=\int d \mathbf{r}\left(\mathcal{H}[\rho(\mathbf{r})]+\frac{\hbar^{2}}{2 m r_{\perp}^{2}} \rho(\mathbf{r})+V_{I}(\mathbf{r}) \rho(\mathbf{r})\right),
$$

where $\rho(\mathbf{r})$ is the He particle density, $\mathcal{H}$ is the OP functional, $V_{I}$ is the helium-impurity potential, and $r_{\perp}$ in the centrifugal term is the distance from the vortex axis. The energy minimization is performed in axial symmetry by mapping the density on a grid of points, putting the vortex line along the $z$ axis and the dopant in the center, at $\mathbf{r}=0$. The numerical code used to evaluate the density profile and energy is the one written for the calculations in $[10]$.

We first consider pure droplets with and without vortex. In Fig. 1 we show density profiles, at $z=0$, obtained for different $N$. For large droplets, the shape approaches that of a rectilinear vortex in the uniform liquid [17]; the core radius is of the order of $1-2 \AA$ and the density oscillates as a consequence of the He-He interaction. In Fig. 2 we plot the energy associated with the vortex flow, defined as

$$
\Delta E_{\mathrm{V}}(N) \equiv E_{\mathrm{V}}(N)-E(N),
$$

where $E_{\mathrm{V}}$ and $E$ are the energies of droplets with and without vortex, respectively. The solid line represents the results obtained with a liquid-drop formula:

$$
\Delta E_{\mathrm{V}}(N)=\alpha N^{1 / 3}+\beta N^{1 / 3} \log N+\gamma N^{-1 / 3},
$$

with the parameters $\alpha=2.868 \mathrm{~K}, \beta=1.445 \mathrm{~K}$, and $\gamma=0.313 \mathrm{~K}$ extracted from a fit to the density functional calculations. This formula works well and the reason can be understood by considering a hollow-core model for the

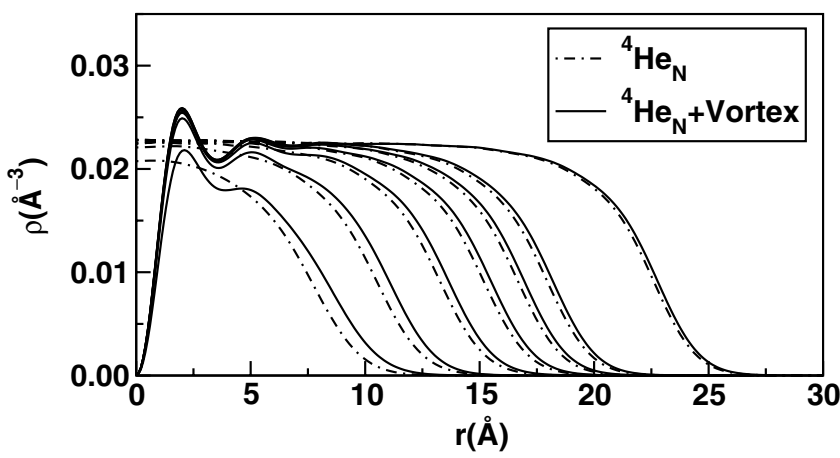

FIG. 1. He density profiles (solid lines) in the $z=0$ plane of drops with $N=40,100,200,300,400,500$, and 1000 having a vortex line along the $z$ axis. The density profiles of vortex-free droplets are also shown (dashed lines). vortex, having core radius $a$, in a droplet of radius $R$ and constant density $\rho_{0}$. Indeed, integrating the kinetic energy of the vortex flow in the limit $R \gg a$, one gets

$$
E_{\mathrm{kin}}=\frac{2 \pi \hbar^{2} \rho_{0}}{m}\left[R \log \left(\frac{2 R}{a}\right)-R+\frac{a^{2}}{4 R}\right] .
$$

Writing $R=r_{0} N^{1 / 3}$ with $r_{0}=\left[3 /\left(4 \pi \rho_{0}\right)\right]^{1 / 3}$, one recovers the $N$ dependence as in Eq. (3). It is worth stressing, however, that this model makes only the fitting formula (3) plausible, but cannot be used to quantitatively relate the best fit parameters $\alpha, \beta$, and $\gamma$ to the model parameters $a$ and $r_{0}$. In fact, Eq. (4) gives only the kinetic energy of a hollow-core vortex in a large droplet, and not the difference between the energies of a droplet with and without vortex. Moreover, the actual core has some structure, the droplet density is not constant, and the core radius is not always much larger than the droplet radius.

The next step is the inclusion of a dopant atom or molecule. As an example, in Fig. 3 we show the He density distribution for a drop of $N=500$ with $\mathrm{HCN}$ hosted in the vortex core. Both the axis of the linear molecule and that of the vortex are taken along $z$. The density is very inhomogeneous near the dopant, due to the complexity of the HCN-He interaction. The energetics of the system can be conveniently analyzed by introducing the following energies:

$$
\begin{gathered}
\Delta E_{\mathrm{V}}^{\mathrm{X}}(N) \equiv E_{\mathrm{X}+\mathrm{V}}(N)-E_{\mathrm{X}}(N), \\
S_{\mathrm{X}}(N) \equiv E_{\mathrm{X}}(N)-E(N), \\
S_{\mathrm{X}+\mathrm{V}}(N) \equiv E_{\mathrm{X}+\mathrm{V}}(N)-E(N),
\end{gathered}
$$

where the subscripts $\mathrm{X}$ and $\mathrm{V}$ refer to drops doped with impurity $\mathrm{X}$ and/or vortex line.

The energy $\Delta E_{\mathrm{V}}^{\mathrm{X}}$ is the one associated with the vortex flow in the doped cluster. In Fig. 2 it is compared with the vortex energy in pure droplets, $\Delta E_{\mathrm{V}}$. The difference,

$$
\delta_{\mathrm{X}}(N)=\Delta E_{\mathrm{V}}^{\mathrm{X}}-\Delta E_{\mathrm{V}}<0,
$$

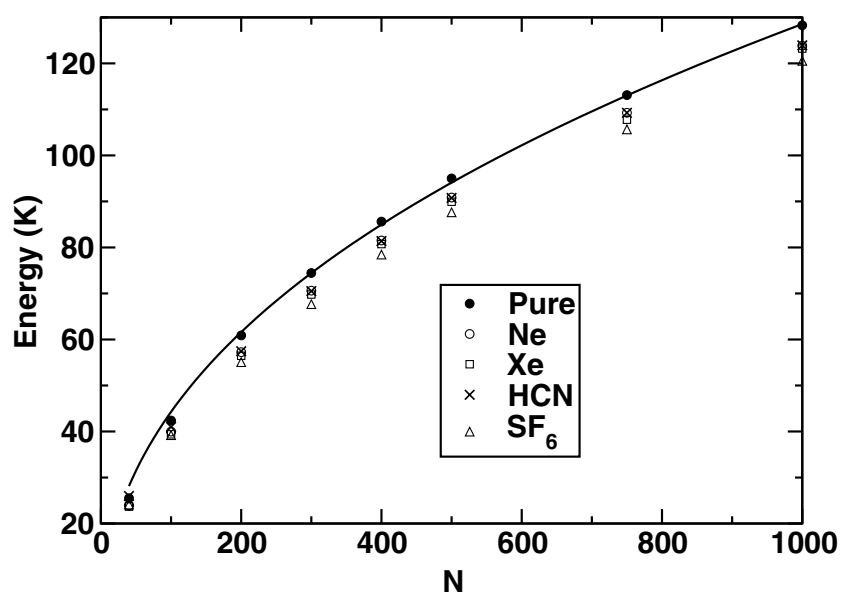

FIG. 2. The vortex energy $\Delta E_{\mathrm{V}}(N)$ (dots) in pure ${ }^{4} \mathrm{He}_{N}$ drops. The line is a fit obtained using Eq. (3). Open symbols are the vortex energies $\Delta E_{\mathrm{V}}^{\mathrm{X}}(N)$ in doped droplets. 


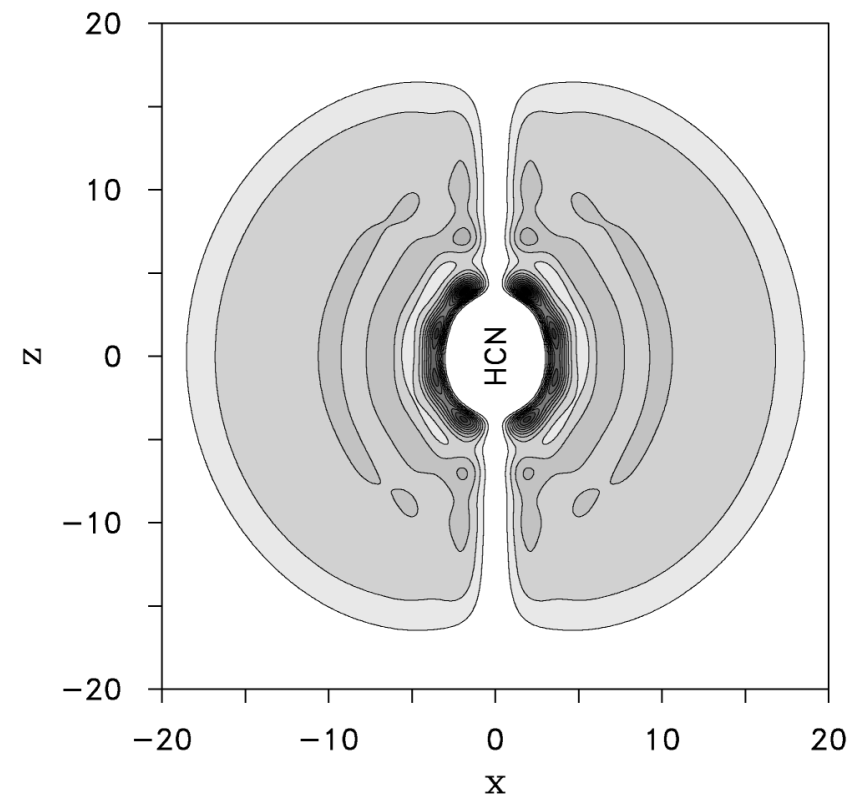

FIG. 3. Density distribution in the $x z$ plane for a $\mathrm{HCN}-\mathrm{He}_{500}$ droplet hosting a vortex along the $z$ axis. Lengths are in $\AA$. Contour lines are drawn between 12 equally spaced intervals of density, where white is for density less than $7.5 \times 10^{-3} \AA^{-3}$, and the darkest level corresponds to density higher than $9 \times$ $10^{-2} \AA^{-3}$.

is almost independent of $N$, apart from the smallest droplets. The reason is that this difference has to do with the "geometrical extension" of the dopant, i.e., the "hole" made by the dopant in the vortex flow, as well as with the distortion of the density near the dopant caused by the pinning of the vortex core. Both effects are localized near the dopant and thus, they are expected to give a shift in energy which becomes $N$ independent for large droplets.

The quantity $S_{\mathrm{X}}(N)$ in Eq. (6) is the solvation energy of the dopant in a vortex-free droplet. The results obtained for $\mathrm{Ne}, \mathrm{Xe}, \mathrm{HCN}$, and $\mathrm{SF}_{6}$ are shown in Fig. 4. As already discussed in [16,22], the solvation energy becomes almost $N$ independent for $N$ larger than a few hundreds. The value at $N=1000$ can be safely taken to represent the solvation energy in the bulk, $S_{\mathrm{X}}(\infty) \simeq S_{\mathrm{X}}(1000)$. For our analysis, we have chosen impurities having binding energies on a wide range.

The key quantity in the present study is the solvation energy of the dopant + vortex complex given by $S_{\mathrm{X}+\mathrm{V}}(N)$ in Eq. (7). The results are shown in Fig. 5. From the definitions (5)-(8), one can also write

$$
\begin{aligned}
S_{\mathrm{X}+\mathrm{V}}(N) & =E_{\mathrm{X}}(N)+\Delta E_{\mathrm{V}}^{\mathrm{X}}(N)-E(N) \\
& =S_{\mathrm{X}}(N)+\Delta E_{\mathrm{V}}(N)+\delta_{\mathrm{X}}(N) .
\end{aligned}
$$

In Fig. 5 we compare $S_{\mathrm{X}+\mathrm{V}}$ with the sum $S_{\mathrm{X}}+\Delta E_{\mathrm{V}}$; the difference is $\delta_{\mathrm{X}}$. The simple picture which emerges from this analysis is that the solvation energy of the dopant + vortex complex is just the sum of the solvation energy of the dopant with no vortex and the extra energy of a vortex in a pure droplet, apart from a small shift which

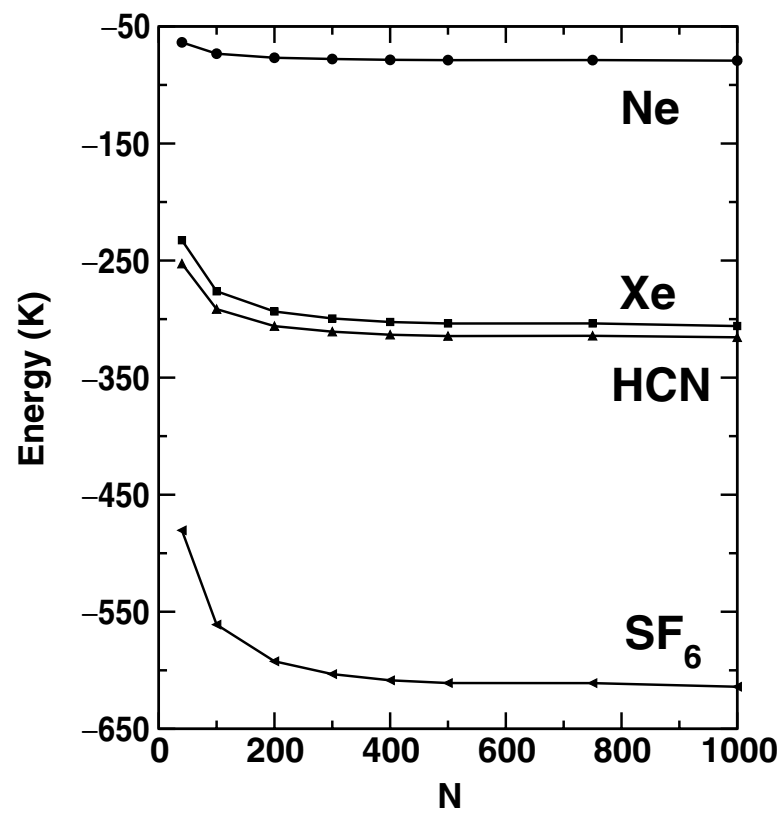

FIG. 4. Solvation energy $S_{\mathrm{X}}(N)$ for $\mathrm{X}=\mathrm{Ne}, \mathrm{Xe}, \mathrm{SF}_{6}$, and HCN. The lines have been drawn to guide the eye.

depends on the dopant. Deviations from this rule are significant only for small droplets, having radius of the order of the size of the dopant. Our results provide a quantitative basis for this picture and typical estimates of $\delta_{\mathrm{X}}$. We also notice that, by rearranging the terms in Eq. (8), this quantity can be written as the difference between the solvation energies of the dopant in a droplet with and without

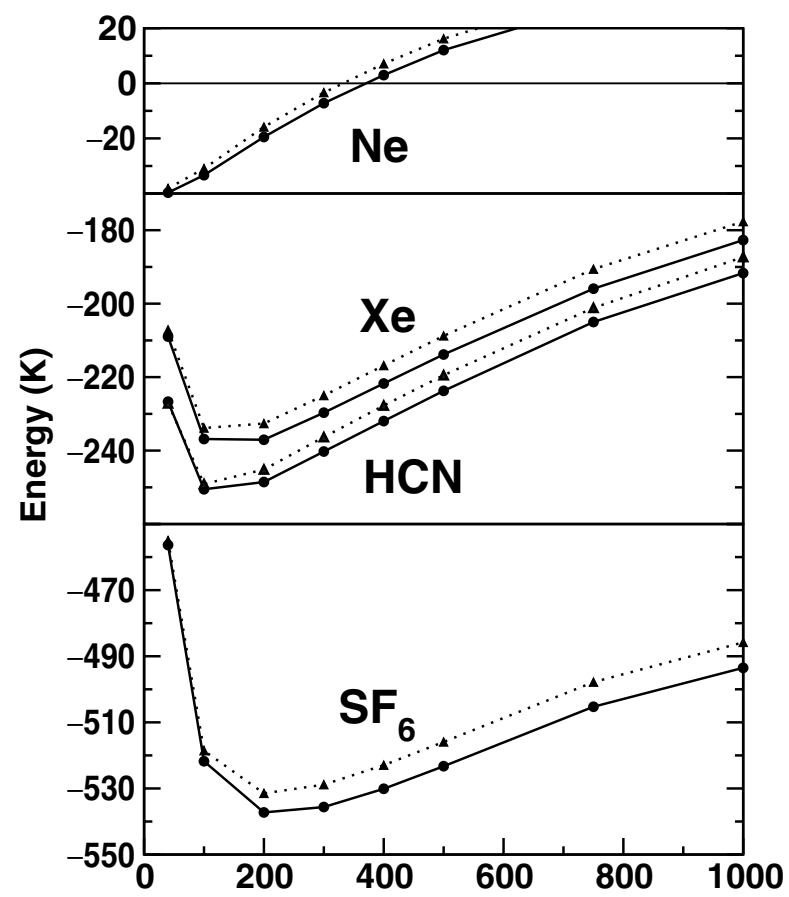

FIG. 5. Excess energies $S_{\mathrm{X}+\mathrm{V}}(N)$ (dots) for $\mathrm{Ne}, \mathrm{Xe}, \mathrm{SF}_{6}$, and HCN. The triangles represent $S_{\mathrm{X}}(N)+\Delta E_{\mathrm{V}}(N)$. Lines have been drawn to guide the eye. 
vortex, $\delta_{\mathrm{X}}=\left[\left(E_{\mathrm{X}+\mathrm{V}}-E_{\mathrm{V}}\right)-\left(E_{\mathrm{X}}-E\right)\right]$, and can, hence, be interpreted as the binding energy of the dopant to the vortex [23].

Since the solvation energy $S_{\mathrm{X}}$ is negative and almost constant for $N>300$ while the vortex energy $\Delta E_{\mathrm{V}}$ always increases, the dopant + vortex complex has a solvation energy which changes sign at some $N_{\text {cr }}$. This means that, for $N<N_{\mathrm{cr}}$, the dopant + vortex complex is energetically favored. In the case of $\mathrm{Ne}$, as one can see in Fig. $5, N_{\text {cr }} \sim 380$. This number is rather small as compared to the typical droplet size in current experiments, and is a consequence of the weak binding of Ne. Dopants with stronger binding have larger $N_{\mathrm{cr}}$. An estimate for $\mathrm{HCN}, \mathrm{Xe}$, and $\mathrm{SF}_{6}$ can be easily obtained by means of the liquid-drop formula. One has to insert expression (3) in Eq. (9) and use the large- $N$ values of $S_{\mathrm{X}}$ and $\delta_{\mathrm{X}}$. The $S_{\mathrm{X}}$ values turn out to be $-310,-320$, and $-622 \mathrm{~K}$, and the $\delta_{\mathrm{X}}$ values are $-5.0,-4.4$, and $-7.7 \mathrm{~K}$ for $\mathrm{Xe}, \mathrm{HCN}$, and $\mathrm{SF}_{6}$, respectively. These numbers yield $N_{\mathrm{cr}} \simeq 7600$ for $\mathrm{Xe}, \simeq 8100$ for $\mathrm{HCN}$, and $\simeq 40000$ for $\mathrm{SF}_{6}$.

In conclusion, the analysis of the energetics of doped helium droplets has allowed us to disclose a possible mechanism to create and stabilize vortex lines. A dopant + vortex complex could be formed by picking up the impurity, assuming that the collision imparts sufficient angular momentum. The vortex line is expected to appear attached to the dopant, since the binding energy $\delta_{\mathrm{X}}$ is negative. The formation of the complex is energetically favored below a critical $N$ which is well within the range of droplet sizes met in current experiments if the dopant has a large solvation energy. A metastable state could also exist for $N>N_{\text {cr }}$, but estimating its lifetime is a more demanding task. One should also explore the energy barrier associated with other possible decay processes. Further work is planned in this direction.

We thank Kevin Lehmann for useful discussions. This work has been performed under Grants No. PB98-1247 from DGESIC, Spain, and No. 1998SGR-00011 from Generalitat of Catalunya.
[1] S. Goyal, D. L. Schutt, and G. Scoles, Phys. Rev. Lett. 69, 933 (1992).

[2] M. Hartmann, R. E. Miller, J. P. Toennies, and A. F. Vilesov, Phys. Rev. Lett. 75, 1566 (1995).

[3] K. B. Whaley, Advances in Molecular Vibrations and Collision Dynamics (JAI Press, New York, 1998), Vol. 3, p. 397.

[4] J. P. Toennies and A.F. Vilesov, Annu. Rev. Phys. Chem. 49, 1 (1998).

[5] K. K. Lehmann and G. Scoles, Science 279, 2065 (1998).

[6] S. Grebenev, J. P. Toennies, and A. F. Vilesov, Science 279, 2083 (1998).

[7] Ph. Sindzingre, M. L. Klein, and D. M. Ceperley, Phys. Rev. Lett. 63, 1601 (1989).

[8] M. Pi, R. Mayol, and M. Barranco, Phys. Rev. Lett. 82, 3093 (1999).

[9] M. Hartmann et al., Phys. Rev. Lett. 76, 4560 (1996).

[10] C. Callegari et al., Phys. Rev. Lett. 83, 5058 (1999); 84, 1848(E) (2000).

[11] C. Raman et al., Phys. Rev. Lett. 83, 2502 (1999).

[12] S. Stringari, Phys. Rev. Lett. 76, 1405 (1996); D. GueryOdelin and S. Stringari, Phys. Rev. Lett. 83, 4452 (1999).

[13] O. M. Maragó et al., Phys. Rev. Lett. 84, 2056 (2000).

[14] M. R. Matthews et al., Phys. Rev. Lett. 83, 2498 (1999); K. W. Madison et al., Phys. Rev. Lett. 84, 806 (2000).

[15] J. Dupont-Roc et al., J. Low Temp. Phys. 81, 31 (1990).

[16] F. Dalfovo, Z. Phys. D 29, 61 (1994).

[17] F. Dalfovo, Phys. Rev. B 46, 5482 (1992).

[18] M. Sadd, G. V. Chester, and L. Reatto, Phys. Rev. Lett. 79, 2490 (1997).

[19] K. T. Tang and J. P. Toennies, Z. Phys. D 1, 91 (1986).

[20] R. T. Pack, E. Piper, G. A. Pfeffer, and J.P. Toennies, J. Chem. Phys. 80, 4940 (1984).

[21] K. M. Atkins and J. M. Hutson, J. Chem. Phys. 105, 440 (1996).

[22] S. M. Gatica, E. S. Hernández, and M. Barranco, J. Chem. Phys. 107, 927 (1997).

[23] The binding energy of ${ }^{3} \mathrm{He}$ atoms and charged ions to a vortex in bulk ${ }^{4} \mathrm{He}$ has been recently calculated by M. Sadd, G. V. Chester, and F. Pederiva, Phys. Rev. Lett. 83, 5310 (1999). 